Research Paper

\title{
Protein 4.1B Suppresses Tumor Metastasis by Regulating Epithelial-mesenchymal Transition Progression in Melanoma Cells
}

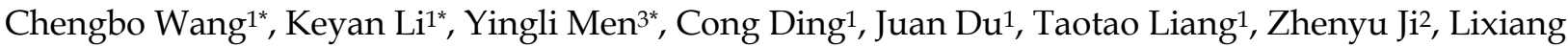 \\ Chen ${ }^{1}$, Ting Wang ${ }^{1 凶}$, Qiaozhen Kang ${ }^{1 凶}$ \\ 1. School of Life Sciences, Zhengzhou University, No. 100 Science Avenue, Zhengzhou 450001, P.R. China \\ 2. Henan Academy of Medical and Pharmaceutical Sciences, Zhengzhou University, 40 University Road, Zhengzhou 450052, P.R. China \\ 3. Translational medical center, People's Hospital of Zhengzhou, 33 Huanghe Road, Zhengzhou 450003, P.R.China \\ *The authors contributed equally to this work. \\ $\bowtie$ Corresponding authors: Prof. Qiaozhen Kang , qzkang@zzu.edu.cn and Dr. Ting Wang, tingwang@zzu.edu.cn; Tel: +8637167783236; Fax: +8637167783235 \\ (C) Ivyspring International Publisher. This is an open access article distributed under the terms of the Creative Commons Attribution (CC BY-NC) license \\ (https://creativecommons.org/licenses/by-nc/4.0/). See http://ivyspring.com/terms for full terms and conditions.
}

Received: 2018.05.22; Accepted: 2019.02.06; Published: 2019.04.16

\begin{abstract}
Epithelial-mesenchymal transition (EMT), which involves the dramatic reorganization of the cytoskeleton, is a crucial initiating step in tumor invasion and metastasis. Protein 4.1B is a membrane-cytoskeleton cross-linker that plays an important role in tumor progression and metastasis; however, the functional roles of 4.1B in melanoma remain unclear. In this study, we aimed to investigate the effect and underlying mechanism of 4.1B on melanoma cells. Our results demonstrated that 4.1B expression was downregulated in murine B16 and B16-F10 melanoma cell lines. Ectopic 4.1B expression significantly inhibited the migration of melanoma cells and pulmonary metastasis. We further investigated the possible mechanism underlying the effect of 4.1B on EMT. The results showed that ectopic 4.1B expression altered the expression of representative EMT markers (E-cadherin, vimentin and $\mathrm{N}$-cadherin), and inhibited the expression of three important transcription factors (Slug, Snail, and Twist) related to EMT in melanoma cells. Moreover, the expression of integrin $\alpha 5, \beta 3$ and matrix metalloproteinase 9 (MMP-9), which is known to regulate cell adhesion, migration and invasion, were suppressed. In conclusion, our data indicate that 4.1B is an important regulator during EMT progression in melanoma cells, which may present a potential target for the prevention and treatment of melanoma.
\end{abstract}

Key words: protein 4.1B, melanoma, migration, epithelial-mesenchymal transition (EMT)

\section{Introduction}

Melanoma is the most lethal skin cancer, and it's incidence has increased at a rate of approximately $3-5 \%$ each year $(1,2)$. Innumerable therapeutic strategies have been designed to treat melanoma, however, no current treatments are able to substantially extended patient survival time $(3,4)$. Recently, the immunotherapy against melanoma have been gradually developed with the elucidation of the role of immune checkpoint molecules in tumor, such as anti-CTLA4 and anti-PD1 agents, nevertheless, many melanoma patients which treated with these agents do not have an objective response (5). The high mortality rate of melanoma is reported to be closely related to tumor metastasis, thus, dissecting the molecular mechanisms underlying melanoma inva- sion and metastasis is urgent for developing novel and more effective therapeutic approaches for this malignancy.

Growing evidences have demonstrated that epithelial-mesenchymal transition (EMT) promotes melanoma cell invasion and metastasis and involves the reorganization of the cytoskeleton (6). During EMT, proteins that interact with the cytoskeleton such as epithelial cadherin (E-cadherin) are downregulated and the expression of mesenchyme markers, including vimentin and neural cadherin $(\mathrm{N}$-cadherin) are upregulated (7-8). Moreover, in the last stage of EMT, the basement membrane and extracellular matrix (ECM) are degraded by the increased expression of proteases (9), such as the matrix metalloproteinases 
(MMPs).

Protein $4.1 \mathrm{~B}$ is one member of membrane cytoskeleton protein 4.1 family (10). Protein 4.1B located under the cell membrane connecting the plasma membrane and the cytoskeleton and participates in the organization of the cytoskeleton (11). Consequently, protein $4.1 \mathrm{~B}$ is regarded as a functional adaptor molecule plays important roles in cell morphology, cell-cell adhesion, and cell migration. More and more studies indicated a tumor suppressor DAL-1, which deleted in adenocarcinoma of the lung, was proved to be a paralogue of $4.1 \mathrm{~B}$ (EPB41L3) (12), and the important function of $4.1 \mathrm{~B}$ in cancer progression is suggested by its frequent loss in a number of cancer (13-17). Moreover, it was demonstrated that protein 4.1B associates with cell motility and adhesion contribute to the process of cancer metastasis by binding various cell adhesion molecules, such as CD44, TSLC-1, Caspr and Caspr2 (18-19). And, our previous study has proved the absent expression of 4.1B in murine melanoma cells (20). However, studies on the biological effects of 4.1B on melanoma cells and the corresponding underlying mechanisms are still lacking. In this study, we aimed to investigate the effect of 4.1B on melanoma cells and EMT. Our results showed that the expression of $4.1 \mathrm{~B}$ was downregulated in melanoma cells. Furthermore, our findings implicated 4.1B as an EMT/metastasis suppressor.

\section{Materials and Methods}

\section{Cell culture}

The mouse melanoma cell lines B16 and B16-F10, as well as mouse embryonic fibroblasts (MEFs), were obtained from the Shanghai Cell Bank of the Chinese Academy of Sciences (SCAS; Shanghai, China). B16 is the primary cell line and the B16-F1 cell line was produced from the mouse model of pulmonary metastasis with intravenous injection of B16 cells, eventually, the B16-F10 cell line was selected repeatedly ten times. Cells were cultured in Dulbecco's modified eagle's medium (DMEM) (Gibco, Grand Island, NY, USA) supplemented with $10 \%$ fetal bovine serum (FBS), 1\% glutamine, and $1 \%$ penicillin-streptomycin at $37^{\circ} \mathrm{C}$ with $5 \% \mathrm{CO}_{2}$.

\section{Plasmids and transfection}

The plasmid pEGFP-N1-4.1B, containing the full-length $4.1 \mathrm{~B}$ coding region, was constructed by our laboratory. The plasmid sequence was verified by the Suzhou Genewiz Biological Technology Co., Ltd (Suzhou, China). The control vector pEGFP-N1 was purchased from Invitrogen (Carlsbad, CA, USA). $5 \times 105$ cells/well were seeded in a 6-well plate and cultured for $20 \mathrm{~h}$ until they reached $70-90 \%$ confluence, then the cells were transfected with $3.5 \mu \mathrm{g} /$ well of plasmid along with $12 \mu \mathrm{l} /$ well Lipofectamine 2000 Transfection Reagent (Invitrogen Life Technologies, Carlsbad, CA, USA) .

\section{RNA extraction and quantitative real-time (qRT)-PCR}

Total RNA was extracted from melanoma cells using TRIzol reagent (CWBIO, Beijing, China) according to the manufacturer's instructions. First-strand cDNA was synthesized from $1 \mu \mathrm{g}$ of total RNA using a PrimeScript ${ }^{\mathrm{TM}}$ RT reagent kit with gDNA Eraser (Perfect Real Time, Takara Bio Inc., Tokyo, Japan). The $25-\mu 1$ reaction mixture included $2 \mu \mathrm{l}$ cDNA $(50 \mu \mathrm{g} / \mu \mathrm{l})$, $1 \mu$ of each primer (Genewiz Biological Technology Co., Ltd), $12.5 \mu 12 \times$ UltraSYBR Mixture (CWBIO), and

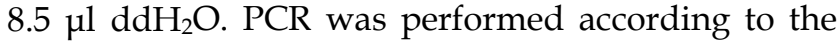
manufacturer's instructions using the Rotor Gene 3000 real-time system (Corbett Robotics Inc., San Francisco, CA, USA) with the following cycling conditions: $95^{\circ} \mathrm{C}$ for $10 \mathrm{~min}$, followed by 40 cycles of $95^{\circ} \mathrm{C}$ for $5 \mathrm{sec}$ and $60^{\circ} \mathrm{C}$ for $1 \mathrm{~min}$. GAPDH was used as an internal control. Relative quantification wasperformed using the $2^{-\Delta \Delta \mathrm{Ct}}$ method. Primer sequences for the target genes are listed in Table I.

Table I. Primers used in qRT-PCR.

\begin{tabular}{lll}
\hline Target gene & Forward primer & Reverse primer \\
\hline Snail & 5'-ACCACTATGCCGCGCTC & 5'-GGTCGTAGGGCTGCTGG \\
& TT-3' & AA-3' \\
Slug & 5'-TCAGCTCAGGAGCAGAC & 5'-ATCCGGAAAGAGGAGA \\
& AGC-3' & GAGG-3' \\
\multirow{2}{*}{ Twist 2} & 5'-TCAGCTAGCCGTGTTTC & 5'-TATTGTTCCTGGGTGTGG \\
& $-3^{\prime}$ & -3' \\
TGF- $\beta 1$ & 5'-GCTAATGGTGGACCGCA & 5'-GCACTGCTTCCCGAATG \\
& ACAAC-3' & TCTG-3' \\
TGF- $\beta 2$ & 5'-CTTCGACGTGACAGACG & 5'-GCAGGGGCAGTGTAAAC \\
& CT-3' & TTATT-3' \\
TGF- $\beta 3$ & 5'-CCTGGCCCTGCTGAACT & 5'-TTGATGTGGCCGAAGTC \\
& TG-3' & CAAC-3' \\
Integrin $\beta 1$ & 5'-TCCCAGCCAGTCCCAAG & 5'-TGCCTCCCAACACGCCC \\
& TGCCATG-3' & CTCATTG-3' \\
GAPDH & 5'-TTCACCACCATGGAGAA & 5'-GGCATGGACTGTGGTCA \\
& GGC-3' & TGA-3' \\
\hline
\end{tabular}

\section{Wound-healing assay}

A nick was made by scraping the middle of the cells with a sterilized tip, when the cells reached $80 \%$ confluence in 6-well plate, and photomicrographs were taken after $24 \mathrm{~h}$. The wound widths in the microscopic images were measured by Image J (National Institutes of Health, Bethesda, MD, USA). This experiment performed in culture media without FBS.

\section{Subcutaneous tumor and pulmonary metastasis animal model}

Five- to six-week-old female C57BL/ 6 mice were purchased from Vital River (Beijing, China). Mice were bred under specific pathogen-free conditions, 
provided sterilized food and water, and housed in a barrier facility with a 12-h light/dark cycle. Mice were randomly divided into three groups, which were pEGFP-N1, pEGFP-N1-4.1B, or control B16. $1 \times 10^{6}$ cells along with $200 \mu \mathrm{l}$ phosphate-buffered saline (PBS) were injected into the subcutaneous right forelimb armpit of each mouse to establish the subcutaneous tumor model. The length and width of the tumor were gauged with vernier caliper every two days and the volume of the tumor was calculated as follows: $V=0.5 \times a \times b^{2}$, where $a$ is the length and $b$ is the width). On day 14, mice were sacrificed and tumors were carefully taken out. The tumors weight was recorded and tumor growth inhibition rates (IRs) were calculated as follows: $I R=(1-$ Average tumor weight of experimental group/Average tumor weight of control group) $\times 100 \%$. To establish the pulmonary metastasis model, a total of $2 \times 10^{5}$ cells along with 200 $\mu \mathrm{l}$ PBS were injected into each mouse via tail vein. On day 14, mice were sacrificed, and the pulmonary melanoma nodules were measured.

\section{Western blot analysis}

Proteins were extracted from MEF, B16, and B16-F10 cells using RIPA lysis buffer containing protease inhibitors (LEYBOLD Cable, Beijing, China). The protein concentration was measured with a BCA kit (LEYBOLD Cable). A total of $20 \mu \mathrm{g}$ proteins were loaded in each lane and then were carried out with $10 \%$ sodium dodecyl sulfate (SDS)-polyacrylamide gel electrophoresis and electroblotting. And then the proteins were blocked with $5 \%$ nonfat milk at room temperature for $2 \mathrm{~h}$ and then incubated with primary antibodies overnight at $4^{\circ} \mathrm{C}$. The antibodies used were as follows: anti-4.1B from the New York Blood Center (New York, NY, USA) and anti-EGFP, anti-E-cadherin, anti-vimentin, anti-N-cadherin, antib-MMP2, antiMMP9, anti-integrin a5 and anti-integrin $\beta 3$ and anti-GAPDH from Bogoo Biotechnology (Shanghai, China). Subsequently, the blots were incubated with horseradish peroxidase (HRP)-conjugated secondary antibody (Bogoo Biotechnology) diluted (1:4000) in blocking buffer for $1 \mathrm{~h}$ at room temperature. Proteins were visualized using an electrochemiluminescence (ECL) detection system (Thermo Fisher Scientific, Rockford, IL, USA).

\section{Flow cytometric analysis}

Cells were isolated by $0.05 \%$ trypsin (LEYBOLD Cable, Beijing, China) and washed twice in 0.5\% bovine serum albumin (BSA). Next, $1 \times 10^{6}$ cells were suspended in $100 \mu 10.5 \%$ BSA and incubated with $2 \mu 1$ rat anti-mouse CD29 antibody or $1.5 \mu \mathrm{l}$ integrin $\beta 1$ antibody followed by incubation with $2 \mu 1$ PE-conjugated anti-rat IgG secondary antibody (30 min each at $4^{\circ} \mathrm{C}$ ). Cells were examined by LSRFortessa Cell Analyzer (BD Biosciences, Franklin Lakes, NJ, USA), and the data were analyzed with FlowJo software (TreeStar, Ashland, OR, USA).

\section{Statistical analysis}

Each experiment was performed in triplicate. Statistical analyses were conducted using GraphPad Prism 5.0 for Windows. The statistical significance of differences was analyzed using an analysis of variance (ANOVA) and Dunnett's post hoc test. A value of $P<0.05$ was considered to indicate statistical significance.

\section{Results}

\subsection{B expression is downregulated in melanoma cell lines}

Earlier studies indicated that $4.1 \mathrm{~B}$ is downregulated or silenced in many cancer cell lines. To investigate the physiological functions of $4.1 \mathrm{~B}$ in melanoma, we analyzed its expression in two immortalized mouse melanoma cell lines, B16 and B16-F10. In conformity with the previous results, our results showed there was no protein expression of 4.1B in B16 and B16-F10 melanoma cells (Figure 1A). Moreover, we demonstrated the absent expression of $4.1 \mathrm{~B}$ in mRNA level in three human melanoma cell lines. (Supplementary Figure 1) To examine the possible roles of $4.1 \mathrm{~B}$ in melanoma cells, we overexpressed protein $4.1 \mathrm{~B}$ by transfecting melanoma cells with the recombinant plasmid pEGFP-N1-4.1B, using pEGFP-N1 as a negative control plasmid. Observations of transfected B16 and B16-F10 cells under a fluorescence microscope (Figure 1B) and western blotting analyses revealed that pEGFPN1-4.1B treatment successfully upregulated protein 4.1B expression (Figure 1C) in both cell lines.

\section{Protein 4.1B suppresses migration of $B 16$ and B 16-F 10 cells}

As cell migration is considered a critical step in metastasis, a wound-healing assay was used to examine the effect of protein $4.1 \mathrm{~B}$ expression on cell migration in vitro. We observed that the edges of wounds in B16 (Figure 2A) and B16-F10 (Figure 2B) cells transfected with $4.1 \mathrm{~B}$ were markedly closer than those in control cells.

\section{Protein 4.1B suppresses melanoma tumor growth and pulmonary metastasis}

Our previous study showed that protein 4.1B inhibits the proliferation of B16 and B16-F10 cells. To confirm the effect of protein 4.1B on tumor growth, B16 cells transfected with pEGFP-N1-4.1B and pEGFP-N1, and non-transfected B16 cells were 
subcutaneously injected into the right forelimb armpits of experimental mice. Tumor size was measured and recorded every two days. As showed in Figure $3 \mathrm{~A}$, the volumes of tumors in mice injected with pEGFP-N1-4.1B B16 cells were much lower than those in mice injected with pEGFP-N1 B16 or non-transfected B16 cells. The average tumor volume in mice in the pEGFP-N1-4.1B group was $500 \mathrm{~mm}^{3}$ at
13 days, while that in the pEGFP-N1 group was 2000 $\mathrm{mm}^{3}$. Moreover, compared with the two control groups, tumor weight was significantly lower in mice injected with pEGFP-N1-4.1B B16 cells (Figure 3B). The tumor inhibition rate was $74.67 \%$ in the pEGFPN1-4.1B group and only $3.49 \%$ in the pEGFP-N1 group (Figure 3C).

\section{A}

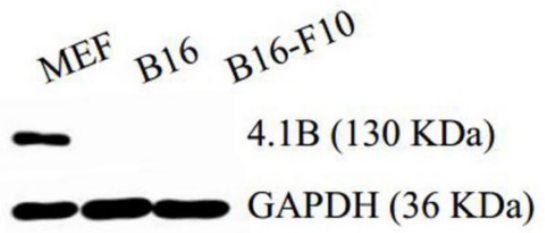

C

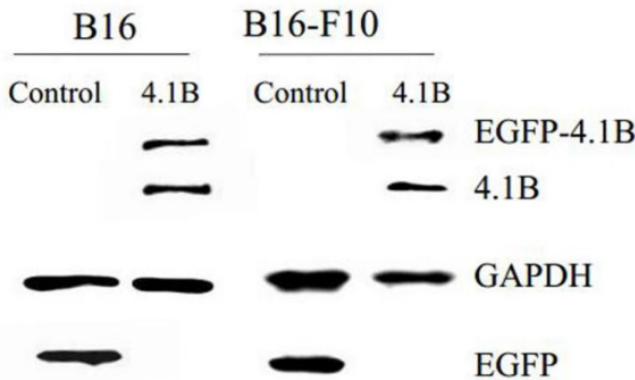

B

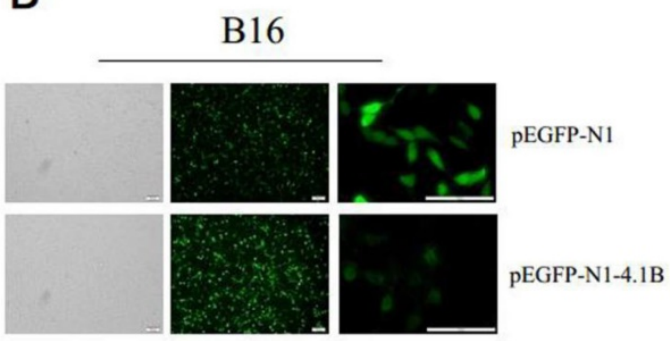

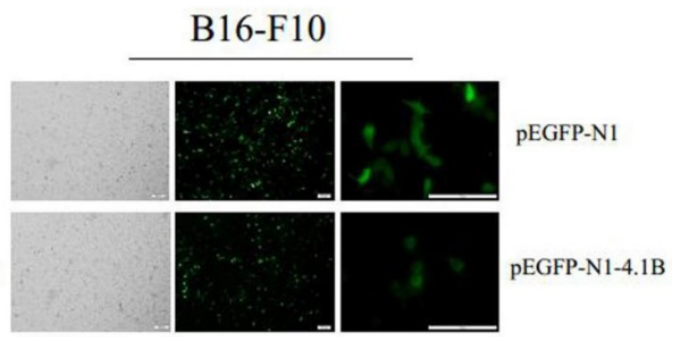

Figure 1. Efficiency of pEGFP-N1-4.1B transfection in B16 and B16-F10 cells. (A) Expression of protein 4.1B in B16 and B16-F10 cells, as analyzed by western blotting. (B) Representative images of B16 and B16-F10 cells transfected with pEGFP-N1 or pEGFP-N1-4.1B. Scale bar: $100 \mu \mathrm{m}$. (C) Expression of 4.1B in B16 and B16-F10 cells transfected with PEGFP-NI-4.1B, as analyzed by western blotting.

A

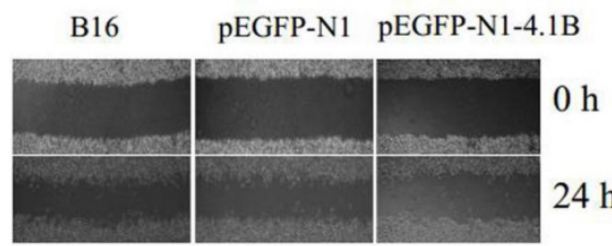

B

B16-F10

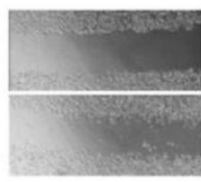

pEGFP-N1-4.1B
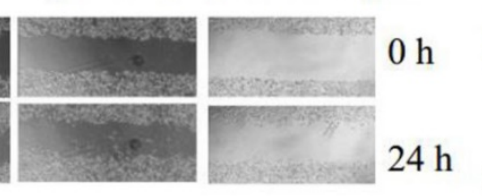

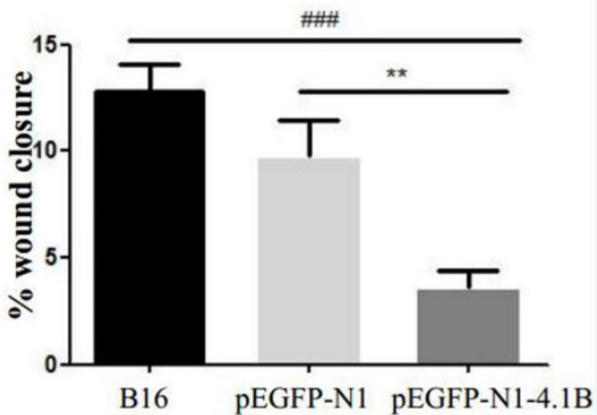

Figure 2. Protein 4.1B suppresses the migration of melanoma cells in vitro. Wound-healing assay in B16 (A) and B16-F10 (B) cells transfected with pEGFP-N1 and PEGFP-N1-4.1B; photographs were obtained at the indicated time points after the scratch was generated (magnification 10x). Quantification is shown on the right in each panel. Values are expressed as mean \pm SEM. $* P<0.05$, $* * P<0.01$, pEGFP-N1-4.1B vs. PEGFP-N1; \#P<0.05, 

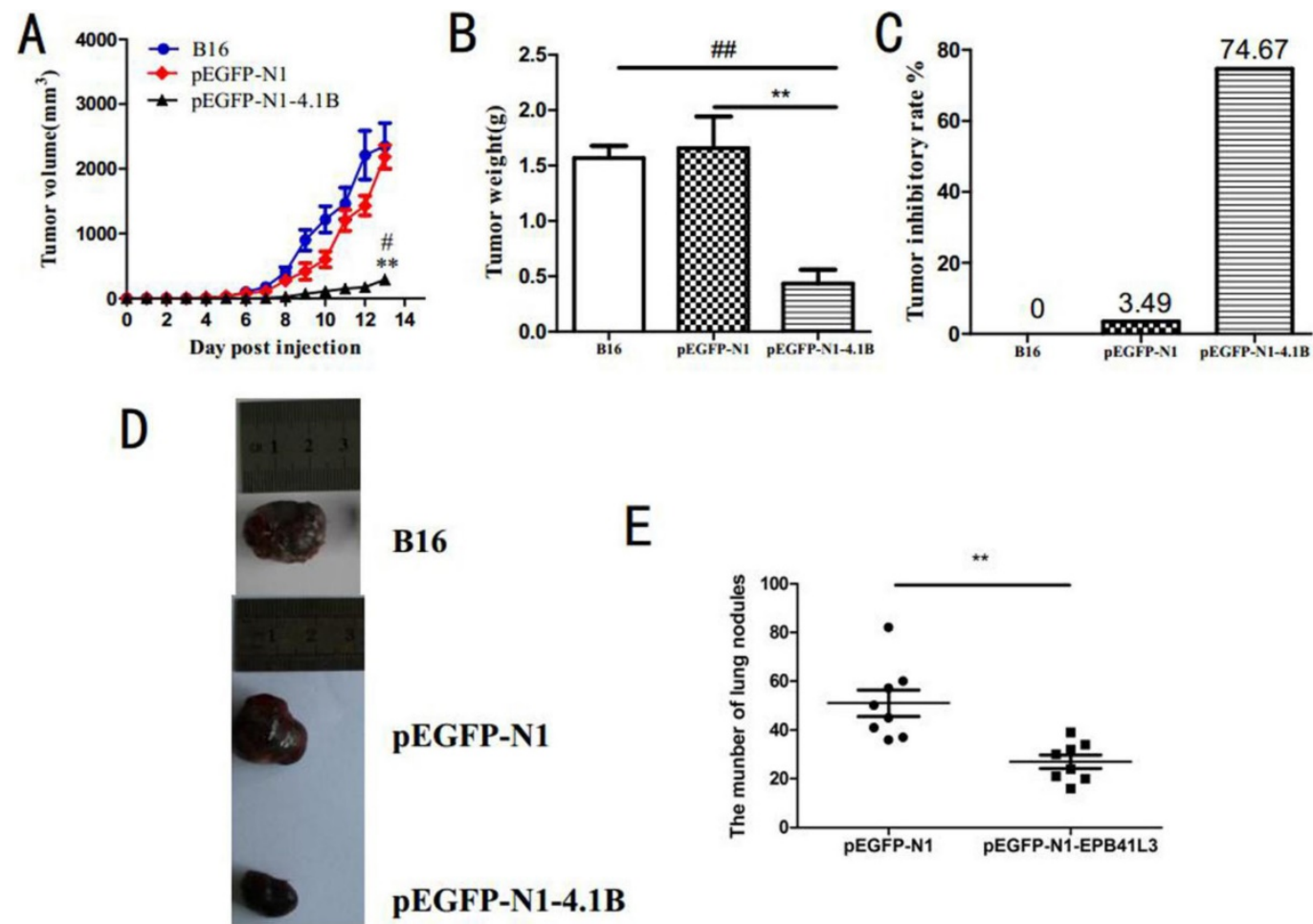

B16

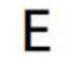

pEGFP-N1

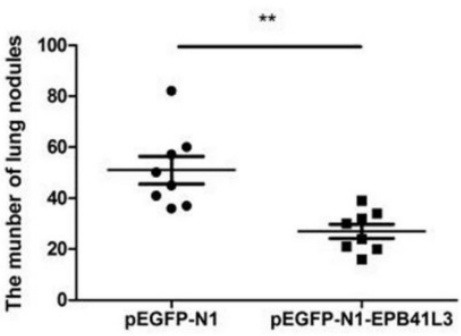

pEGFP-N1-4.1B

Figure 3. Protein 4.1B attenuates melanoma cell growth and metastasis in vivo. B16, B16-pEGFP-N1 and B16-pEGFP-N1-4.1B cells were used to inoculate the right forelimb by subcutaneous injection. B16-F10-pEGFP-N1 and B16-F10-pEGFP-N1-4.1B cells were injected into the tail vein. Tumor volume (A), tumor weight (B), tumor inhibition rate (C), number of lung nodules (E). (D) Representative images of the tumors in each group.

In addition, a model of pulmonary metastasis was established through the tail vein injection of pEGFP-N1-4.1B or pEGFP-N1 B16-F10 cells into mice. All mice were sacrificed 14 days after injection. As shown in Figure 3E, more metastatic nodules were found in the lungs of the mice injected with pEGFP-N1-4.1B-transfected cells than in the lungs of those injected with pEGFP-N1-transfected cells. This suggests that protein $4.1 \mathrm{~B}$ inhibits the pulmonary metastasis of melanoma. Combined with the above findings, we conclude that protein 4.1B suppresses melanoma tumor growth and metastasis.

\subsection{B overexpression alters the expression of EMT markers in B16 and B16-F10 cells}

It has been shown that EMT is closely associated with tumor invasion and metastasis. Therefore, we next investigated the effect of $4.1 \mathrm{~B}$ on the EMT phenotype in B16 and B16-F10 cells. Western blotting results showed that, compared with transfection with empty vector, transfection with $4.1 \mathrm{~B}$ markedly upregulated the expression of E-cadherin and downregulated the expression of $\mathrm{N}$-cadherin and vimentin in B16 and B16-F10 cells (Figure 4A). In addition, the expression of the EMT-related transcription factors Snail, Slug, and Twist2 was significantly downregulated at the mRNA level in B16 and B16-F10 cells transfected with 4.1B (Figure 4B, $4 C)$. Transforming growth factor- $\beta$ (TGF- $\beta$ ) family proteins paly predominant role in the induction of EMT. To determine whether $4.1 \mathrm{~B}$ is associated with the TGF- $\beta$ signaling pathway in melanoma, we used fluorescent quantitative PCR to detect the expression of TGF- $\beta 1$, TGF- $\beta 2$, and TGF- $\beta 3$ in B16 and B16-F10 cells transfected with 4.1B. As shown in Figure 5D and $5 \mathrm{E}$, the expression of TGF- $\beta 1$ and TGF- $\beta 2$ was not significantly affected by $4.1 \mathrm{~B}$ expression. However, the expression of TGF- $\beta 3$ was downregulated in 4.1B-transfected B16 and B16-F10 cells compared with levels in control cells. These results suggest that protein 4.1B suppresses EMT in B16 and B16-F10 cells, likely by affecting the TGF- $\beta 3$ signaling pathway.

\section{Protein 4.1B alters the expression of integrins and matrix metalloproteinases in $B 16$ and B 16-F 10 cells}

Recent studies have suggested that the expression of certain integrins is closely related with cell invasion and migration, and many integrins with low expression in normal epithelial tissues are up-regulated during EMT. Thus, we explored the effect of protein $4.1 \mathrm{~B}$ on integrin $\beta 1, \beta 3$ and $\alpha 5$ expression. As showed in Figure $5 \mathrm{~A}-\mathrm{C}$, there was no difference in the expression of total integrin $\beta 1$ or active integrin $\beta 1$ between 4.1B-transfected and control plasmid-transfected B16 and B16-F10 cells (Figure 5A-B), nevertheless, overexpression of 4.1B suppressed the expression of integrin $\beta 3$ and $\alpha 5$ 
(Figure 5C). Matrix metalloproteinases (MMPs) degrade the basement membrane and ECM to regulate cell migration and invasion. To further confirm the mechanism by which cell migration is inhibited following the overexpression of 4.1B, MMP-9 and MMP-2 expression was detected in B16 and B16-F10 cells. The mRNA expression levels of
MMP9 and MMP2 were downregulated in B16 and B16-F10 cells transfected with pEGFP-N1-4.1B. At the protein level, MMP-9 expression was downregulated, while MMP-2 expression was not altered in B16 and B16-F10 cells transfected with pEGFP-N1-4.1B compared to levels in control cells (Figure 5D-F).
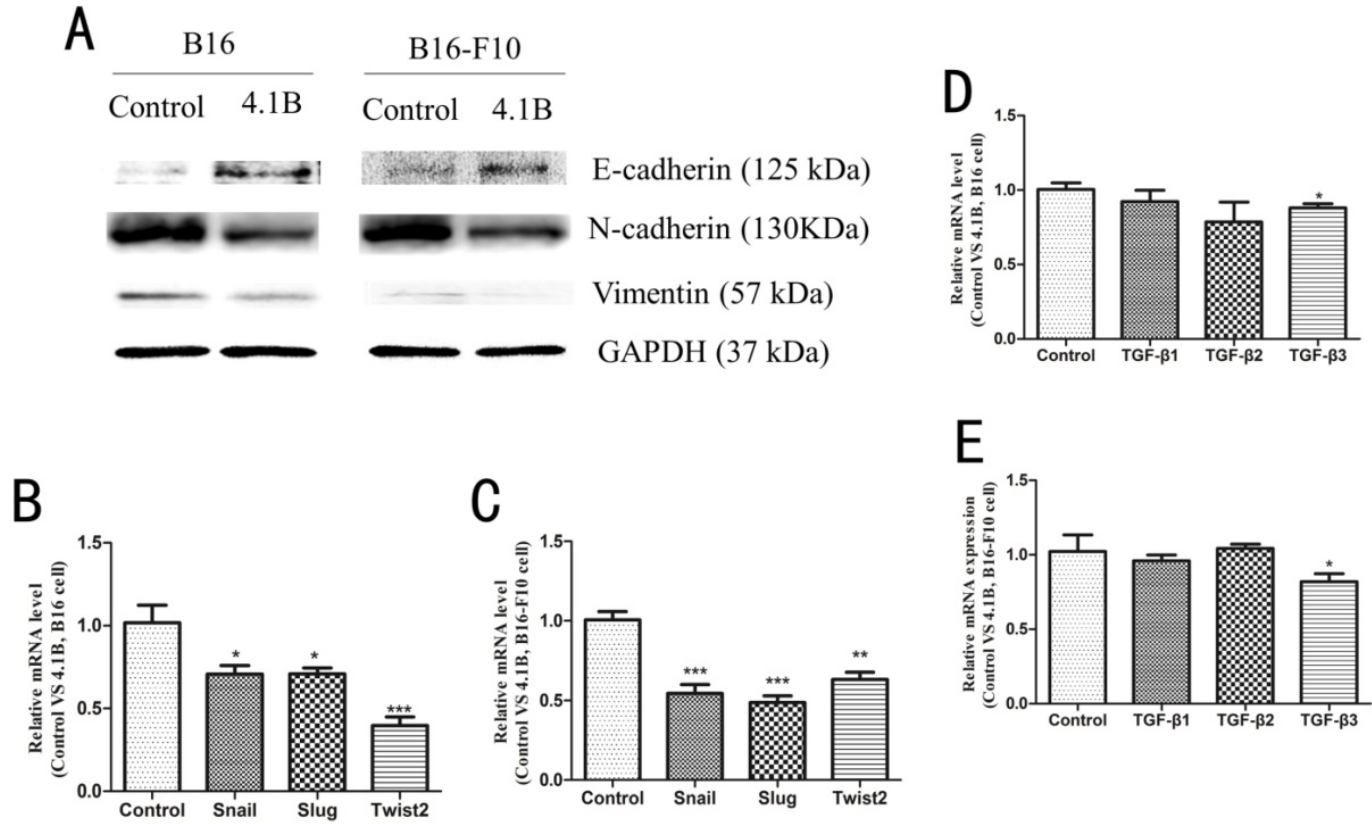

Figure 4. Protein 4.1B expression alters the expression of EMT markers. (A) Protein expression of $\mathrm{N}$-cadherin, E-cadherin and vimentin, as determined by western blotting. mRNA expression levels of EMT-related transcription factors in B16 (B) and B16-F10 (C) cells stably expressing control (pEGFP-NI) or 4.1B (pEGFP-N1-4.1B) plasmids, as determined by qRT-PCR. mRNA expression levels of TGF- $\beta$ family members in B16 (D) and B16-F10 (E) cells stably expressing control (PEGFP-NI) or 4.1B (pEGFP-NI-4.1B) plasmids, as determined by qRT-PCR.
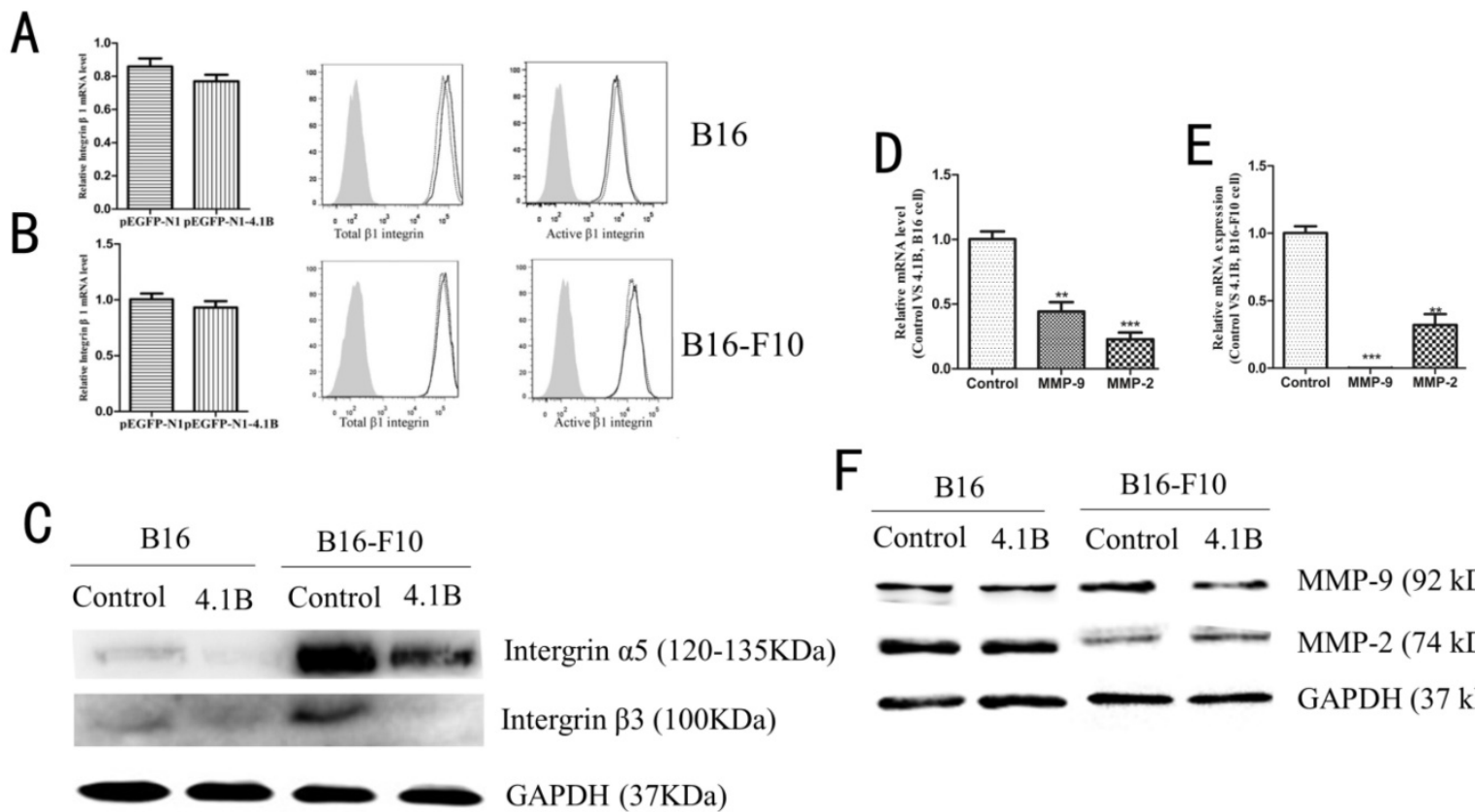

$\mathrm{F}$

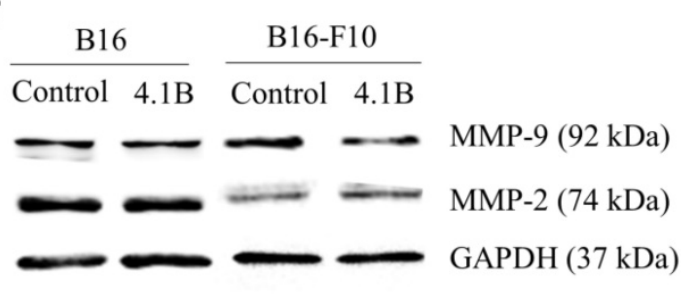

Figure 5. Protein 4.1B downregulated integrins and MMP9 expression. The expression of integrin $\beta 1$ in B16 (A) and B16-F10 (B) cells was determined by qRT-PCR and flow cytometry. (C) The expression of integrin $\alpha 5$ and $\beta 3$ in B16 and B16-F10 cells was determined by western blotting. The mRNA expression levels of MMP9 and MMP2 in B16 (D) and B16-F10 (E) cells stably expressing control (pEGFP-NI) or 4.1B (pEGFP-NI-4.1B) plasmids, as determined by qRT-PCR. (F) Protein expression of MMP-9 and MMP-2, as determined by western blotting. 


\section{Discussion}

As an important component of the membraneassociated cytoskeleton, protein $4.1 \mathrm{~B}$ was originally identified in cell-cell junctions. The SABD and FERM domains of $4.1 \mathrm{~B}$ lead this protein to function as a scaffold by binding to the cell membrane and connecting to the cytoskeleton (21). This specific structure is essential for the maintenance of cell polarity and cell-cell adhesions. Moreover, 4.1B was originally considered a tumor suppressor gene based on its frequent downregulation in several cancers, including cervical cancer, laryngeal squamous cell carcinoma, and breast cancer $(22,23)$. These findings are in agreement with our primary result that $4.1 \mathrm{~B}$ expression was absent in melanoma cell lines. Thus, based on this result, we speculate that 4.1B may inhibit the progression of melanoma. As expected, ectopic $4.1 \mathrm{~B}$ inhibited the cell growth and the metastatic capacity of melanoma cell lines in vivo and in vitro. These results suggest that $4.1 \mathrm{~B}$ acts as an anti-oncoprotein by suppressing the progression of melanoma.

Previous studies have reported that EMT occurs during epithelial tumor progression to increase the motility and invasiveness of cancer cells $(24,25)$. The cell junctions are destructed to initiate an EMT and the proteins associated with cell-cell junctions are degraded, such as E-cadherin at the plasma membrane is cleaved and degraded. The downregulation of cell junction proteins is accompanied by the increased expression of the mesenchymal proteins. Specifically, the repression of E-cadherin is balanced by the upregulation of $\mathrm{N}$-cadherin, which leads to a 'cadherin switch' that breaks down the epithelial cell junctions (26). As 4.1B is associated with cytoskeletal molecules and regulates the cellular functions involved in migration under physiological conditions, we speculated that $4.1 \mathrm{~B}$ might suppress metastasis by inhibiting EMT. In the present study, we observed that the ectopic expression of $4.1 \mathrm{~B}$ upregulated the expression of E-cadherin and downregulated the expression levels of $\mathrm{N}$-cadherin in melanoma cells. In addition, 4.1B expression decreased expression of vimentin, which altered the completeness of cytoskeleton and then also suppressed the EMT in melanoma cells. The alteration that the repression of the epithelial characteristic and activation of the mesenchymal phenotype is mediated by multiple transcription factors, including Snail, Slug and Twist2 (27). Our results demonstrated that ectopic expression of $4.1 \mathrm{~B}$ inhibited the expression of Snail, Slug and Twist2, which supported that ectopic expression of $4.1 \mathrm{~B}$ altered the expression of E-cadherin, N-cadherin and vimentin in melanoma cells.

Upon the progression of EMT, the ECM is remodelled and cell-ECM interactions are changed. Integrins are the transmembrane heterodimeric extracellular matrix receptors that provide the linkage between the ECM proteins and intracellular skeleton proteins. During the progress of EMT, the cells interact with a different ECM, thus, the expressions of some epithelial integrins are suppressed, but the others are activated. It has been proved that increased integrin $\beta 1, \beta 3$ and a5 expression during EMT increases cell adhesion to fibronectin, which is also upregulated and promotes cell invasion and migration in melanoma cell $(28,29)$. Moreover, changes of the integrins expression trigger the increased expression of proteases during EMT, such as MMP2 and MMP9, thus promoting ECM proteins degradation and the cells invasion (30). Here, we found that ectopic expression of $4.1 \mathrm{~B}$ suppressed the expression of integrin a5, $\beta 3$ and MMP-9, however, no effect on integrin $\beta 1$ and MMP2. Together those results suggest that protein $4.1 \mathrm{~B}$ suppresses tumor metastasis by regulating epithelial-mesenchymal transition progression in melanoma cells.

According to EMT-based studies, more and more evidence has indicated that multiple signaling pathways are involved in the progress of EMT, including the Wnt, Notch, TGF- $\beta$, fibroblast growth factor pathways and epidermal growth factor (31). Among these, TGF- $\beta$ signal plays a predominant role in the induction of EMT. TGF- $\beta$ regulates the expression of three important EMT-related transcription factors (Snail, ZEB1/2, and Twist) through initiating Smad signaling (32). And then Snail indirectly effects on other transcription factors, such as Sp-1 and Ets-1, which are considered to be partially responsible for the activation of MMP-9 (33). In this study, we found that the expression of TGF $\beta 3$ was significantly downregulated at the mRNA level in melanoma cells transfected with 4.1B. This suggests that protein 4.1B suppresses the progression of EMT in melanoma cells, probably through the TGF- $\beta$ signaling pathway.

In conclusion, this is the first report demonstrating that the overexpression of $4.1 \mathrm{~B}$ inhibits cell proliferation, migration, and EMT in melanoma cells. Thus, 4.1B may represent a novel molecular target for the prevention and treatment of melanoma.

\section{Supplementary Material}

Supplementary figures.

http://www.medsci.org/v16p0529s1.pdf

\section{Acknowledgments}

This work was supported by grants from the National Natural Science Foundation of China (81602 537, 81571526 and 8167154) and Natural Science Foundation of Henan Province of China (162300410277). 


\section{Competing Interests}

The authors have declared that no competing interest exists.

\section{References}

1. Mylene S, Veilleux MD, Neil H, et al. Biologics in patients with skin diseases. Allergy Clin Immunol. 2017; 139(5): 1423-1430.

2. Siegel RL, Miller KD, Jemal A. Cancer Statistics 2017. CA Cancer J Clin. 2017; 67(1): 7-30.

3. Spagnolo F, Queirolo P. Upcoming strategies for the treatment of metastatic melanoma. Arch Dermatol Res. 2012; 304(3): 177-84.

4. Olszanski A J. Current and future roles of targeted therapy and immunotherapy in advanced melanoma. J Manag Care Spec Pharm. 2014; 20(4): 346-56.

5. Postow M A, Chesney J, Pavlick A C, et al. Nivolumab and ipilimumab versus ipilimumab in untreated melanoma. N Engl J Med. 2015, 372(21):2006-2017.

6. Yang J, Weinberg R A. Epithelial-mesenchymal transition: at the crossroads of development and tumor metastasis. Developmental Cell. 2008; 14(6): 818-829.

7. Tam WL, Weinberg RA. The epigenetics of epithelial-mesenchymal plasticity in cancer. Nature Medicine. 2013; 19(11): 1438-1449.

8. Savagner P. The epithelial-mesenchymal transition (EMT) Phenomenon. Annals of Oncology Official Journal of the European Society for Medical Oncology. 2010; 21(7): 89-92.

9. Gilles C, Newgreen D F, Sato H, et al. Matrix Metalloproteases and Epithelial-to-Mesenchymal Transition. Rise and Fall of Epithelial Phenotype. Springer US. 2005:297-315.

10. Bretscher A, Edwards K, Fehon R G. ERM proteins and merlin: integrators at the cell cortex. Nature Reviews Molecular Cell Biology. 2002; 3(8): 586.

11. Diakowski W, Grzybek M, Sikorski A F. Protein 4.1, a component of the erythrocyte membrane skeleton and its related homologue proteins forming the protein 4.1/FERM superfamily. 2006, 44(4):231-48.

12. Tran YK, Bogler O, Gorse KM, WIELAND I, NEWSHAM IF. A novel member of the NF2/ERM/4.1 superfamily with growth suppressing properties in lung cancer. Cancer Res. 1999; 59(1): 35-43.

13. Steenbergen RD, Kramer D, Braakhuis BJ, et al. TSLC1 gene silencing in cervical cancer cell lines and cervicalneoplasia. J Natl Cancer Inst. 2004; 96(4): 294-305.

14. Lu B, Di W, Wang H, et al. Tumor suppressor TSLC1 is implicated in cell proliferation, invasion and apoptosis in laryngeal squamous cell carcinoma byregulating Akt signaling pathway. Tumour Biol. 2012; 33(6): 2007-2017.

15. Heller G, Geradts J, Ziegler B, et al. Downregulation of TSLC1 and DAL-1 expression occurs frequently in breast cancer. Breast Cancer Res Treat. 2007; 103(3): 283-291.

16. Ito T, Shimada $Y$, Hashimoto $Y$, et al. Involvement of TSLC1 in progression of esophageal squamous cell carcinoma. Cancer Res 2003; 63(19): 6320-6326.

17. Kikuchi S, Yamada d, Fukami T, et al. Promoter methylation of DAL-1/4.1B predicts poor prognosis in non-small cell lung cancer. Clin Cancer Res. 2005; 11(8): 2954-2961

18. Busam RD, Thorsell AG, Flores A, et al. Structural basis of tumor suppressor in lung cancer 1 (TSLC1) binding to differentially expressed in adenocarcinoma of the lung (DAL-1/4.1B). J Biol Chem. 2011; 286(6): $4511-4516$.

19. Charboneau AL, Singh V, Yu T, NEWSHAM IF. Suppression of growth and increased cellular attachment after expression of DAL-1 in MCF-7 breast cancer cells. Int J Cancer. 2002; 100: 181-188.

20. Men Y, Kang Q, Ding C, et al. Expression of protein 4.1 family in melanoma cell lines and its effect on cell proliferation. Journal of Southern Medical University. 2016; 36(5): 649

21. Haase D, Meister M, Muley T. FRMD3, a novel putative tumour suppressor in NSCLC. Oncogene. 2007; 26: 4464-4468.

22. Bernkopf D B, Williams E D. Potential role of EPB41L3 (protein 4.1B/Dal-1) as a target for treatment of advanced prostate cancer. Expert Opin Ther Targets. 2008; 12(7): 845-853.

23. Cavanna T, Pokorna E P, Gray C. Evidence for protein $4.1 \mathrm{~B}$ acting as a metastasis suppressor. Journal of Cell Science. 2007; 120(4): 606-616.

24. Andriani F, Bertolini G, Facchinetti F, et al. Conversion to stem-cell state in response to microenvironmental cues is egulated by balance betweenepithelial and mesenchymal features in lung cancer cells. Molecular Oncology. 2016; 10(2): 253-271.

25. Beerling E, Seinstra d, Dewit e, et al. Plasticity between epithelial and mesenchymal states unlinks emt from metastasis-enhancing stem cell capacity. Cell Reports. 2016; 14(10): 2281

26. Wheelock M J, Shintani $Y$, Maeda M, et al. Cadherin switching. Journal of Cell Science. 2008, 121(Pt 6):727-735.

27. Lamouille $\mathrm{S}, \mathrm{Xu}$ J, Derynck R. Molecular mechanisms of epithelial-mesenchymal transition. Nature Reviews Molecular Cell Biology. 2014, 15(3):178-96

28. Huang R, Rofstad E K. Integrins as therapeutic targets in the organ-specific metastasis of human malignant melanoma. Journal of Experimental \& Clinical Cancer Research. 2018, 37(1):92
29. Mckenzie J A, Liu T, Jung J Y, et al. Survivin promotion of melanoma metastasis requires upregulation of a5 integrin. Carcinogenesis. 2013, 34(9):2137-44

30. Nisticò P, Bissell M J, Radisky D C. Epithelial-mesenchymal transition: general principles and pathological relevance with special emphasis on the role of matrix metalloproteinases. Cold Spring Harbor Perspectives in Biology. 2012, 4(2):441.

31. Saito A. EMT and EndMT: Regulated in similar ways? J Biochem. 2013; 153: 493-495.

32. Derynck R, Zhang YE. Smad-dependent and Smad-independent pathways in TGF- $\beta$ family signalling. Nature. 2003; 425: 577-584.

33. Jorda M, Olmeda D, Vinyals A. Upregulation of MMP-9 in MDCK epithelial cell line in response to expression of the Snail transcription factor. J Cell Sci. 2005; 118: 3371-3385. 